\title{
212 - Involvement, Worries and Loneliness of Family Caregivers of People with Dementia during the COVID-19 Visitor-ban in Long-term Care Facilities \\ Marleen Prins, Bernadette Willemse, Claudia van der Velden, Anne Margriet Pot \\ \& Henriëtte van der Roest
}

\section{Background}

To prevent COVID-19 from spreading in long-term care facilities (LTCFs), the Dutch government took national restrictive measures, including a visitor-ban in LTCFs between mid-March and May 2020. Physical visits were replaced by alternatives as telephone or video calls. This study examines the relationship between the involvement of family caregivers (informal caregivers, ICs) of people with dementia (PWD) living in LTCFs and IC mental health during the visitor-ban. Furthermore, we examine whether this relationship is moderated by the frequency of contact with PwD during the visitor-ban and resilience of ICs.

\section{Methods}

A cross-sectional study was carried out, 375 Dutch long-term care organizations were invited by email to participate. LTCFs sent eligible ICs a link to an anonymous online survey. Family involvement was assessed by the visiting frequency and doing social (e.g. drinking coffee), or social and task-related (e.g. laundry) activities during visits before the visitor-ban.

\section{Results}

958 ICs of PwD participated. Contact frequency increased for $17 \%$ ICs and decreased for $25 \%$ compared to visiting frequency. $43 \%$ of ICs did only social activities and $57 \%$ social and task-related activities. ICs who visited their relatives at least once a week before the visitor-ban were more worried during the visitor-ban than those with less regular visits (main effect). Contact frequency during the visitor-ban was a moderating factor, ICs who visited the PwD daily before, but had at least weekly contact during the visitor-ban, worried less. No main effects for activity type were found on loneliness, however resilience was a moderating factor. Resilient ICs who did more diverse activities (task and social related) before the visitor-ban, experienced less loneliness during the visitor ban.

\section{Conclusions}

The results implicate that to reduce worries amongst ICs, LTCFs should facilitate in continuing contact with PwD during a visitor-ban, specifically in highly involved ICs. Also, non-resilient ICs that generally only do social activities are more prone to loneliness. It is advisable for healthcare and welfare professionals to reach out to this group, to help them with overcoming their loneliness.

\section{3 - ECT-AD: Ethics and informed consent issues}

Presenter: Louis Nykamp, M.D.

Co-authors:

Brent P. Forester, M.D., M.Sc.

Adriana P. Hermida, M.D.

Martina Mueller, Ph.D.

Georgios Petrides, M.D.

Hannah Heintz, B.A.

Maria I. Lapid, M.D.

Preferred Presentation Type: Free/Oral Communication Presentations 